\title{
SUBLATTICES OF COMPLETE LATTICES WITH CONTINUITY CONDITIONS
}

\author{
FRIEDRICH WEHRUNG \\ Dedicated to the memory of Ivan Rival
}

\begin{abstract}
Various embedding problems of lattices into complete lattices are solved. We prove that for any join-semilattice $S$ with the minimal join-cover refinement property, the ideal lattice $\operatorname{Id} S$ of $S$ is both algebraic and dually algebraic. Furthermore, if there are no infinite $D$-sequences in $\mathrm{J}(S)$, then Id $S$ can be embedded into a direct product of finite lower bounded lattices. We also find a system of infinitary identities that characterize sublattices of complete, lower continuous, and join-semidistributive lattices. These conditions are satisfied by any (not necessarily finitely generated) lower bounded lattice and by any locally finite, join-semidistributive lattice. Furthermore, they imply M. Erné's dual staircase distributivity.

On the other hand, we prove that the subspace lattice of any infinitedimensional vector space cannot be embedded into any $\aleph_{0}$-complete, $\aleph_{0}$-upper continuous, and $\aleph_{0}$-lower continuous lattice. A similar result holds for the lattice of all order-convex subsets of any infinite chain.
\end{abstract}

\section{INTRODUCTION}

It is a classical result that the ideal lattice $\operatorname{Id} L$ of a lattice $L$ is an algebraic lattice, furthermore, it contains an isomorphic copy of $L$ and it satisfies the same identities as $L$, see [13, Lemma I.4.8]. A much harder result is that every modular lattice embeds, within its variety, into an algebraic spatial lattice (see Section 2 for precise definitions), see 14. Say that a lattice is bi-algebraic, if it is both algebraic and dually algebraic. While investigating lattices of convex subsets, the authors of 21, 22] came across the following problem, which is stated as Problem 5 in [21.

Can every lattice be embedded into some bi-algebraic lattice?

After having asked several experts in lattice theory, we finally came to the surprising conclusion that the answer to that question was unknown. In the present paper, we solve this problem in the negative, see Section 12 More specifically, we prove that both the lattice of all subspaces of any infinite-dimensional vector space and the lattice of all order-convex subsets of any infinite chain cannot be embedded into any bi-algebraic lattice, see Corollaries 12.4 and 12.5

Nevertheless, it turns out that one can prove many positive results in this topic that seem to have been unknown until now. We introduce a new class of lattices, the so-called fermentable lattices, see Definition [5.1] The class of fermentable lattices

Date: October 25, 2018.

2000 Mathematics Subject Classification. 06B23, 06B05, 06B15, 06B35, $06 \mathrm{C} 05$.

Key words and phrases. Lattice, complete, ideal, filter, upper continuous, lower continuous, algebraic, dually algebraic, join-dependency, lower bounded, fermentable, dually zipper distributive, dually staircase distributive. 
includes the class of all ideal lattices of finitely generated lower bounded lattices. We give in Theorem 5.2 an alternative proof of a result also established, with a different method, by M. V. Semenova in 20]: Every fermentable lattice can be embedded into a direct product of finite lower bounded lattices. This extends [2, Corollary 2.2], that states that every finitely presented lower bounded lattice embeds into a direct product of finite lower bounded lattices. It also extends the result, established in 1 by using 9, that the ideal lattice of any free lattice embeds into some direct product of finite lower bounded lattices - observe that any direct product of finite lattices is bi-algebraic. Furthermore, we obtain other related results, such as: The ideal lattice of a join-semilattice with the minimal join-cover refinement property is bi-algebraic, see Corollary 6.3 A common extension of lower continuity and join-semidistributivity, called (after M. Erné) dual *-distributivity, is proved for fermentable lattices, see Corollary 7.4 .

However, these results do not extend to the class of all (not necessarily finitely generated) lower bounded lattices, see Section 2 Nevertheless, for those we still obtain partial results, such as the following.

- We find a system of infinitary identities characterizing sublattices of complete, lower continuous, join-semidistributive lattices (see Theorem 11.2). We observe that these 'identities' are satisfied by any lower bounded lattice (see Corollary 11.5) and by any locally finite, join-semidistributive lattice (see Corollary 10.2). Furthermore, they imply M. Erné's "dual staircase distributivity" (see Corollary 11.3).

- A finitely generated lower bounded lattice may not be embeddable into any complete, lower continuous, lower bounded lattice (see Example 11.77).

- There exists a locally finite, lower bounded lattice that cannot be embedded into any complete, upper continuous, join-semidistributive lattice (see Example 11.9).

- A lattice has a complete embedding into some complete, lower continuous, join-semidistributive lattice iff it satisfies M. Erné's "dual *-distributivity" (see Theorem 11.11).

Some of our results are easy extensions of known results, such as the lower continuity result proved in Lemma 6.1 or the dual $*$-distributivity result of Corollary 7.4 - still they do not seem to follow right away from the already existing literature. Some other results of the present paper seem to be completely new, such as our characterization result of sublattices of complete, lower continuous, join-semidistributive lattices (see Theorem 11.2). Some patterns of our proof that certain lattices cannot be embedded into any bi-algebraic lattice (see Section 12) can be found in von Neumann's classical proof that the perspectivity relation in a continuous geometry is transitive, see 17. However, continuous geometries are modular lattices while our negative results can be applied to non-modular lattices such as those in Corollary 12.5. Still, as our results cover both the modular and the join-semidistributive case, putting them in perspective in the present paper seemed to us worth the effort.

\section{BASIC NOTIONS}

For a set $X$, we denote by $X^{<\omega}$ the set of all finite sequences of elements of $X$, and we denote by $\langle\boldsymbol{s}, \boldsymbol{t}\rangle \mapsto \boldsymbol{s} \frown \boldsymbol{t}$ the concatenation of finite sequences. We let $\mathfrak{P}(X)$ denote the powerset of $X$. 
For partially ordered sets $K$ and $L$, a map $f: K \rightarrow L$ is meet-complete, if $x=\bigwedge_{i \in I} x_{i}$ in $K$ implies that $f(x)=\bigwedge_{i \in I} f\left(x_{i}\right)$ in $L$, for all $x \in K$ and every family $\left(x_{i}\right)_{i \in I}$ of elements of $K$; "join-complete" is defined dually. We say that $f$ is complete, if it is both meet-complete and join-complete.

A lattice $L$ is lower continuous, if the equality

$$
a \vee \bigwedge X=\bigwedge(a \vee X),
$$

holds, for any $a \in L$ and any downward directed $X \subseteq L$ such that $\bigwedge X$ exists. Of course, we put $a \vee X=\{a \vee x \mid x \in X\}$. Restricting the cardinality of $X$ to be at most $\kappa$, for $\kappa$ either an infinite cardinal or $\infty$, yields $\kappa$-lower continuity. Upper continuity is defined dually.

A lattice is join-semidistributive, if it satisfies the quasi-identity

$$
x \vee y=x \vee z \Longrightarrow x \vee y=x \vee(y \wedge z) .
$$

For lattices $K$ and $L$, a homomorphism $f: K \rightarrow L$ is lower bounded, if the preimage under $f$ of any principal filter of $L$ is either empty or has a least element. As in 2, a lattice $L$ is lower bounded, if every lattice homomorphism from a finitely generated free lattice to $L$ is lower bounded. Equivalently, every finitely generated sublattice of $L$ is lower bounded in the sense of 8 . It is well-known that every lower bounded lattice is join-semidistributive, see [8, Theorem 2.20].

For a join-semilattice $S$, we put $S^{-}=S \backslash\{0\}$, if $S$ has a zero element, and $S^{-}=S$, otherwise. We denote by $\mathrm{J}(S)$ the set of join-irreducible elements of $S$. We say that a subset $\Sigma$ of $S$ is join-generates $S$ (resp., finitely join-generates) $S$, if every element of $S$ is a join (resp., a finite join) of elements of $\Sigma$.

An element $a \in S$ is compact, if for every upward directed subset $X$ of $S$, if $\bigvee X$ is defined and $a \leq \bigvee X$, then $a \in \downarrow X$. We say that $S$ is algebraic, if it is complete and the set of compact elements of $S$ join-generates $S$. Note that there are other works, such as $[3$, where completeness is not included in the definition of an algebraic lattice.

We say that $S$ is spatial, if the set of all completely join-irreducible elements of $S$ join-generates $S$. It is well known that every dually algebraic lattice is lower continuous - see [5. Lemma 2.3] or [10, Section 1.4], and spatial - see [10, Theorem I.4.22] or [12, Lemma 1.3.2].

For any $X \subseteq S$, we put

$$
\begin{aligned}
& \downarrow X=\{y \in S \mid \exists x \in X \text { such that } y \leq x\} \\
& \uparrow X=\{y \in S \mid \exists x \in X \text { such that } x \leq y\} .
\end{aligned}
$$

We abuse notation slightly by putting $\downarrow x=\downarrow\{x\}$ and $\uparrow x=\uparrow\{x\}$, for all $x \in S$. For subsets $X$ and $Y$ of $S$, we say that $X$ refines $Y$, in notation $X \ll Y$, if $X \subseteq \downarrow Y$.

For $\kappa$ being either a cardinal number or $\infty$, let the prefix " $\kappa$-" mean restriction to families of cardinality at most $\kappa$, for example, a lattice is $\aleph_{0}$-meet-complete, if every countable subset has a meet, while it is $\infty$-meet-complete, if it is meet-complete.

\section{RELATIVIZATIONS OF THE MINIMAL JOIN-COVER REFINEMENT PROPERTY}

Definition 3.1. Let $S$ be a join-semilattice and let $\Sigma \subseteq S$. For an element $a$ of $S^{-}$, we put

- $\mathcal{C}(a)=\{X \subseteq S \mid X$ is finite, $a \notin \downarrow X$, and $a \leq \bigvee X\}$. The elements of $\mathcal{C}(a)$ are called the nontrivial join-covers of $a$. 
- $\mathcal{M}(a)=\{E \in \mathcal{C}(a) \mid \forall X \in \mathcal{C}(a), X \ll E$ implies that $E \subseteq X\}$. The elements of $\mathcal{M}(a)$ are called the minimal nontrivial join-covers of $a$, and we put $\mathcal{M}_{\Sigma}(a)=\mathcal{M}(a) \cap \mathfrak{P}(\Sigma)$.

Furthermore, we introduce the following properties of the pair $\langle S, \Sigma\rangle$ :

- We say that $S$ has the $\Sigma$-weak minimal join-cover refinement property, in short the $\Sigma$-WMCRP, if every element of $\mathcal{C}(p)$ can be refined by an element of $\mathcal{M}_{\Sigma}(p)$, for all $p \in \Sigma$.

- We say that $S$ has the $\Sigma$-minimal join-cover refinement property, in short the $\Sigma-M C R P$, if it has the $\Sigma$-WMCRP and $\mathcal{M}_{\Sigma}(p)$ is finite, for all $p \in \Sigma$.

Of course, the $\Sigma$-MCRP implies the $\Sigma$-WMCRP. Observe that for $a \in S$, every element of $\mathcal{M}_{\Sigma}(a)$ is an antichain of $\Sigma \cap \mathrm{J}(S)$. The classical minimal join-cover refinement property, in short MCRP, see [8], is the $S$-MCRP. Observe that it implies that $\mathrm{J}(S)$ finitely join-generates $S$.

The join-dependency relation $D$ on a join-semilattice $S$ is defined on $\mathrm{J}(S)$ as usual, that is, for $a, b \in \mathrm{J}(S)$, the relation $a D b$ holds if $a \neq b$ and there exists $c \in S$ such that $a \leq b \vee c$ but $a \not \leq x \vee c$ for all $x<b$. Another useful equivalent definition is provided by the following lemma.

Lemma 3.2. Let $S$ be a join-semilattice and let $\Sigma \subseteq \mathrm{J}(S)$. We suppose that $S$ satisfies the $\Sigma$-WMCRP. Then for all $a, b \in \Sigma$, the relation $a D b$ holds iff there exists $E \in \mathcal{M}_{\Sigma}(a)$ such that $b \in E$.

Proof. The proof is virtually the same as the one of [8, Lemma 2.31].

\section{More lattices With the MCRP}

It is well-known that every free lattice has the MCRP, see [8, Chapter II]. Furthermore, every finitely presented lattice has the MCRP as well, see [7. In this section, we present a few easy common extensions of these results.

Definition 4.1. For a poset $P$, we denote by $\mathrm{F}_{\mathbf{L}}(P)$ the free lattice on $P$. This means that $\mathrm{F}_{\mathbf{L}}(P)$ is generated, as a lattice, by (an isomorphic copy of) $P$, and any order-preserving map from $P$ to any lattice $L$ can be extended to a unique lattice homomorphism from $\mathrm{F}_{\mathbf{L}}(P)$ to $L$.

Proposition 4.2. Every lattice of the form $\mathrm{F}_{\mathbf{L}}(P) / \theta$, for a poset $P$ and a finitely generated congruence $\theta$ of $\mathrm{F}_{\mathbf{L}}(P)$, has the $M C R P$.

Proof. Put $L=\mathrm{F}_{\mathbf{L}}(P) / \theta$. We identify $\mathrm{F}_{\mathbf{L}}(Q)$ with its canonical image in $\mathrm{F}_{\mathbf{L}}(P)$, for any subposet $Q$ of $P$.

Since $\theta$ is finitely generated, there are $m<\omega$ and $a_{i}, b_{i}$, for $i<m$, in $\mathrm{F}_{\mathbf{L}}(P)$, such that $\theta=\bigvee_{i<m} \Theta_{\mathrm{F}_{\mathbf{L}}(P)}\left(a_{i}, b_{i}\right)$. Let $Q_{0}$ be a finite subset of $P$ such that $a_{i}$, $b_{i} \in \mathrm{F}_{\mathbf{L}}\left(Q_{0}\right)$, for all $i<m$.

Let $v \in L$. There exists $\dot{v} \in \mathrm{F}_{\mathbf{L}}(P)$ such that $v=[\dot{v}]_{\theta}$. Let $Q \subseteq P$ be finite such that $Q_{0} \subseteq Q$ and $\dot{v} \in \mathrm{F}_{\mathbf{L}}(Q)$. We put $\psi=\bigvee_{i<m} \Theta_{\mathrm{F}_{\mathbf{L}}(Q)}\left(a_{i}, b_{i}\right)$, a finitely generated congruence of $\mathrm{F}_{\mathbf{L}}(Q)$. Put $K=\mathrm{F}_{\mathbf{L}}(Q) / \psi$. There exists a unique lattice homomorphism $f: K \rightarrow L$ such that $f\left([x]_{\psi}\right)=[x]_{\theta}$, for all $x \in \mathrm{F}_{\mathbf{L}}(Q)$.

Put $K^{\circ}=K \cup\{\mathrm{O}\}$ and $L^{\circ}=L \cup\{\mathrm{O}\}$, for a new zero element O. Extend $f$ to a homomorphism from $K^{\circ}$ to $L^{\circ}$ by putting $f(\mathrm{O})=\mathrm{O}$. Since $Q$ is finite, it is possible to define a map $g_{0}: P \rightarrow K^{\circ}$ by the rule

$$
g_{0}(p)=\bigvee\left([q]_{\psi} \mid q \in Q, q \leq p\right) \text {, for all } p \in P,
$$


with the convention that $\bigvee \varnothing=0$. Since $g_{0}$ is order-preserving, it extends to a unique lattice homomorphism $g: \mathrm{F}_{\mathbf{L}}(P) \rightarrow K^{\circ}$. Observe that $g(x)=[x]_{\psi}$, for all $x \in \mathrm{F}_{\mathbf{L}}(Q)$. In particular, $g\left(a_{i}\right)=g\left(b_{i}\right)$, for all $i<m$, thus there exists a unique lattice homomorphism $h: L^{\circ} \rightarrow K^{\circ}$ such that $h(\mathrm{O})=\mathrm{O}$ and $h\left([x]_{\theta}\right)=g(x)$, for all $x \in \mathrm{F}_{\mathbf{L}}(P)$.

Claim. The following assertions hold.

(i) $h f(y)=y$, for all $y \in K^{\circ}$; thus $f$ is an embedding.

(ii) The inequality $f h(y) \leq y$ holds, for all $y \in L^{\circ}$.

Proof of Claim. (i) In the nontrivial case $y \neq 0$, we can write $y=[t]_{\psi}$, for some $t \in \mathrm{F}_{\mathbf{L}}(Q)$. Then $h f(y)=h\left([t]_{\theta}\right)=g(t)=[t]_{\psi}=y$.

(ii) For all $p \in P$, we compute:

$$
f h\left([p]_{\theta}\right)=f g(p)=\bigvee\left(f\left([q]_{\psi}\right) \mid q \leq p \text { in } Q\right)=\bigvee\left([q]_{\theta} \mid q \leq p \text { in } Q\right) \leq[p]_{\theta} .
$$

Since $\left\{[p]_{\theta} \mid p \in P\right\}$ generates $\mathrm{F}_{\mathbf{L}}(P)$, the conclusion follows.

Claim.

Set $u=[\dot{v}]_{\psi}$. Hence $v=f(u)$ and, by (i) of the Claim above, $u=h(v)$. Since $K$ is a finitely presented lattice, it has the MCRP, see [7. Let $I_{l}$, for $l<n$, denote the minimal nontrivial join-covers of $u$ in $K$. Since $v=f(u)$, the set $J_{l}=f\left[I_{l}\right]$ is a nontrivial join-cover of $v$, for all $l<n$. Hence, to conclude the proof, it suffices to establish that every nontrivial join-cover $J$ of $v$ in $L$ is refined by some $J_{l}$. Observe first that $u=h(v) \leq \bigvee h[J]$. If $u \leq h(t)$, for some $t \in J$, then, by (ii) of the Claim above, $v=f(u) \leq f h(t) \leq t$, a contradiction; hence, $h[J]$ is a nontrivial join-cover of $u$ in $K$, thus there exists $l<n$ such that $I_{l} \ll h[J]$, whence $J_{l}=f\left[I_{l}\right] \ll f h[J]$, so, again by (ii) of the Claim above, $J_{l} \ll J$, which concludes the proof.

We observe the following immediate consequence of [8, Lemma 5.3].

Proposition 4.3. Let $f: L \rightarrow K$ be a lower bounded homomorphism of lattices. If $L$ has the MCRP, then so does $K$.

Corollary 4.4. Every lower bounded homomorphic image of $\mathrm{F}_{\mathbf{L}}(P) / \theta$ has the $M C R P$, for any poset $P$ and any finitely generated congruence $\theta$ of $\mathrm{F}_{\mathbf{L}}(P)$.

Definition 4.5. A lattice $L$ is finitely defined, if it is defined by finitely many relations (within the class of all lattices).

It is clear that finitely defined lattices are exactly the quotients of free lattices by finitely generated congruences. Hence, Corollary 4.4 applies to finitely defined lattices.

\section{LEAVENS AND FERMENTABLE LATTICES}

An infinite $D$-sequence of a join-semilattice $L$ is a sequence $\left(a_{n}\right)_{n<\omega}$ of elements of $\mathrm{J}(L)$ such that $a_{n} D a_{n+1}$ for all $n<\omega$.

Definition 5.1. Let $L$ be a join-semilattice. A subset $\Sigma$ of $\mathrm{J}(L)$ is a leaven of $L$, if the following statements hold:

(i) $\Sigma$ join-generates $L$.

(ii) $L$ satisfies the $\Sigma$-MCRP.

(iii) There is no infinite $D$-sequence of elements of $\Sigma$ in $L$.

We say that $L$ is fermentable, if it has a leaven. 
This terminology is inspired from P. Pudlák and J. Tůma's beautiful designation as "finitely fermentable" (see [18) those lattices that are nowadays called "finite lower bounded". In particular, a finite lattice is fermentable iff it is lower bounded.

Every free lattice and every finitely generated lower bounded lattice is fermentable (see [8]). Furthermore, the ideal lattice of the free lattice $\mathrm{F}_{\mathbf{L}}(X)$ on any nonempty set $X$ is, by Corollary 5.4 fermentable, but it is not lower bounded in case $X$ has at least three elements (see 2 ).

The following result that every fermentable lattice can be embedded into a direct product of finite lower bounded lattices is also established by M. V. Semenova in 20].

Theorem 5.2. Every fermentable join-semilattice $L$ has a meet-complete joinembedding into some direct product of finite lower bounded lattices.

Observe that every direct product of finite lower bounded lattices is bi-algebraic and fermentable.

Proof. Let $\Sigma$ be a leaven of $L$. We denote by $\unlhd$ the reflexive, transitive closure of the join-dependency relation on $\Sigma$ (see Lemma 3.2), and we put

$$
\Sigma_{p}=\{q \in \Sigma \mid p \unlhd q\}, \text { for all } p \in \Sigma .
$$

Claim 1. The set $\Sigma_{p}$ is finite, for all $p \in \Sigma$.

Proof of Claim. Define $T$ as the set of all finite sequences $\left\langle p_{0}, p_{1}, \ldots, p_{n}\right\rangle$ of elements of $\Sigma$ such that $p_{i} D p_{i+1}$, for all $i<n$. Then $T$, endowed with the initial segment ordering, is a tree. Furthermore, since $L$ satisfies the $\Sigma$-MCRP, $T$ is finitely branching. Since there is no infinite $D$-sequence in $\Sigma$, the tree $T$ has no infinite branch, thus, by König's Theorem, every connected component of $T$ is finite. The conclusion follows immediately.

Claim 1.

For any $p \in \Sigma$, denote by $L_{p}$ the set of all joins of elements of $\Sigma_{p}$, with a new zero element $\mathrm{O}$ added as the join of the empty set.

Claim 2. The lattice $L_{p}$ is finite lower bounded, for all $p \in \Sigma$.

Proof of Claim. The finiteness of $L_{p}$ follows from Claim 1 Moreover, $\Sigma_{p}$ is contained in $\mathrm{J}(L) \cap L_{p}$, thus in $\mathrm{J}\left(L_{p}\right)$. Since every element of $L_{p}$ is a join of elements of $\Sigma_{p}$, it follows that $\mathrm{J}\left(L_{p}\right)=\Sigma_{p}$.

For all $q, r \in \Sigma_{p}$, the relations $q D_{L_{p}} r$ and $q D_{L} r$ are equivalent. Thus, $L_{p}$ does not have $D$-cycles. Since $L_{p}$ is finite, it is lower bounded.

Claim 2.

We put $\Phi_{p}(x)=\downarrow x \cap \Sigma_{p}$ and $\varphi_{p}(x)=\bigvee \Phi_{p}(x)$, for all $p \in \Sigma$ and $x \in L$. In particular, $\varphi_{p}$ is a map from $L$ to $L_{p}$. It is obvious that $q \leq x$ iff $q \leq \varphi_{p}(x)$, for all $q \in \Sigma_{p}$ and all $x \in L$; hence $\varphi_{p}$ is meet-complete. Let $a, b \in L$ and let $q \in \Sigma_{p}$ with $q \leq \varphi_{p}(a \vee b)$, we prove that $q \leq \varphi_{p}(a) \vee \varphi_{p}(b)$. This is obvious if either $q \leq a$ or $q \leq b$, so suppose that $q \not \leq a, b$. Since $q \leq a \vee b$, there exists $I \in \mathcal{M}_{\Sigma}(q)$ such that $I \ll\{a, b\}$. From $I \in \mathcal{M}_{\Sigma}(q)$ it follows that $I \subseteq \Sigma_{p}$, thus, since $I \ll\{a, b\}$, the relation $I \subseteq \Phi_{p}(a) \cup \Phi_{p}(b)$ holds, whence $q \leq \bigvee I \leq \varphi_{p}(a) \vee \varphi_{p}(b)$. It follows that $\varphi_{p}$ is a join-homomorphism from $L$ to $L_{p}$.

Hence, the map $\varphi: L \rightarrow \prod_{p \in \Sigma} L_{p}$ defined by the rule $\varphi(x)=\left(\varphi_{p}(x)\right)_{p \in \Sigma}$ is a join-homomorphism, and it is meet-complete. Furthermore, for $a, b \in L$ such that $a \not \leq b$, there exists $p \in \Sigma$ such that $p \leq a$ and $p \not \leq b$, thus $p \leq \varphi_{p}(a)$ while $p \not \leq \varphi_{p}(b)$; whence $\varphi(a) \not \leq \varphi(b)$. Therefore, $\varphi$ is an order-embedding. 
Remark 5.3. The proof above shows, in fact, that all the lattice homomorphisms $\varphi_{p}: L \rightarrow L_{p}$, for $p \in \Sigma$, are lower bounded.

Every finitely generated lower bounded lattice $L$ is fermentable, with leaven $\mathrm{J}(L)$ (see [8. Theorem 2.38]); hence, by Theorem [5.2 it embeds into a direct product of finite lower bounded lattices. Therefore, every finitely generated lower bounded lattice belongs to the quasivariety $\mathbf{Q}\left(\mathcal{L} \mathcal{B}_{\text {fin }}\right)$ generated by all finite lower bounded lattices. As every quasivariety is closed under direct limits, this gives another proof of the result, first established in 2. Theorem 2.1], that every lower bounded lattice belongs to $\mathbf{Q}\left(\mathcal{L B}_{\text {fin }}\right)$.

Corollary 5.4. Let $S$ be a join-semilattice with the MCRP and no infinite Dsequence of join-irreducible elements. Then the ideal lattice $\operatorname{Id} S$ is fermentable; thus it embeds into a direct product of finite lower bounded lattices.

Proof. It is straightforward to verify that $\Sigma=\{\downarrow p \mid p \in \mathrm{J}(S)\}$ is a leaven of $L=$ Id $S$; whence $L$ is fermentable. The conclusion follows immediately from Theorem 5.2

In particular, it follows from Corollary 5.4 that $\operatorname{Id} L$ is fermentable, for every lattice $L$ which is either free or finitely generated lower bounded. It cannot be extended to arbitrary lower bounded lattices, for any direct product of finite lower bounded lattices is complete, upper continuous, and join-semidistributive, while, on the other hand, the (locally finite, lower bounded) lattice of Example 11.9 cannot be embedded into any complete, upper continuous, join-semidistributive lattice. Furthermore, by Example 11.10 Corollary 5.4 cannot be extended to the filter lattice Fil $L$ of $L$.

\section{LOWER CONTINUITY}

Our main lemma is the following, very similar in statement and in proof to 8 Theorem 2.25].

Lemma 6.1. Let $L$ be a join-semilattice and let $\Sigma$ be a join-generating subset of $\mathrm{J}(L)$ such that $L$ has the $\Sigma-M C R P$. Then $L$ is lower continuous.

Proof. Let $a \in L$ and let $X \subseteq L$ be a downward directed subset admitting a meet, $b=\bigwedge X$. We prove the equality $a \vee b=\bigwedge(a \vee X)$. Since $\Sigma$ join-generates $S$, it suffices to prove that for any $p \in \Sigma$ such that $p \leq a \vee x$ for all $x \in X$, the inequality $p \leq a \vee b$ holds. This is trivial in case either $p \leq a$ or $p \leq x$ for all $x \in X$, so suppose that this does not occur; let $x_{0} \in X$ such that $p \not \leq x_{0}$, and put $X^{\prime}=\left\{x \in X \mid x \leq x_{0}\right\}$. We put $\mu(x)=\left\{E \in \mathcal{M}_{\Sigma}(p) \mid E \ll\{a, x\}\right\}$, for all $x \in X^{\prime}$. Observe that $\mu(x) \neq \varnothing$ (because of the $\Sigma$-MCRP) and that $x \leq y$ implies that $\mu(x) \subseteq \mu(y)$, for all $x \leq y$ in $X^{\prime}$. Since $\mathcal{M}_{\Sigma}(p)$ is finite (because of the $\Sigma$-MCRP), the intersection of all $\mu(x)$, for $x \in X^{\prime}$, is nonempty; pick an element $E$ in this set. Since $E \ll\{a, x\}$, for all $x \in X^{\prime}$, the relation $E \ll\{a, b\}$ holds, whence $p \leq \bigvee E \leq a \vee b$.

The following lemma is folklore.

Lemma 6.2. Every algebraic and lower continuous lattice is dually algebraic. 
Proof. Let $L$ be an algebraic and lower continuous lattice. Since $L$ is dually spatial (see [10, Theorem I.4.22]), it suffices to prove that every completely meet-irreducible element $u$ of $L$ is dually compact. Let $X$ be a downward directed subset of $L$ such that $\bigwedge X \leq u$. Suppose that $x \not \leq u$ for all $x \in X$. So $u^{*}$, the unique upper cover of $u$, lies below $u \vee x$, for all $x \in X$; whence, by the lower continuity of $L$, $u^{*} \leq u \vee \wedge X=u$, a contradiction. Hence, $u$ is dually compact.

Corollary 6.3. Let $S$ be a join-semilattice satisfying the MCRP. Then the ideal lattice Id $S$ of $S$ is bi-algebraic.

Proof. Apply Lemmas 6.1 and 6.2 to $L=\operatorname{Id} S$ and $\Sigma=\{\downarrow p \mid p \in \mathrm{J}(S)\}$.

Corollary 6.4. Let $P$ be a poset, let $\theta$ be a finitely generated congruence of $\mathrm{F}_{\mathbf{L}}(P)$, and let $L$ be a lower bounded homomorphic image of $\mathrm{F}_{\mathbf{L}}(P) / \theta$. Then $\operatorname{Id} L$ is bialgebraic.

In particular, we obtain the following corollary.

Corollary 6.5. Let $L$ be a lower bounded homomorphic image of a finitely defined lattice. Then Id $L$ is bi-algebraic.

Corollary 6.5 cannot be extended to the class of all lower bounded lattices (see Section [2]. In fact, Corollary [6.5] does not even extend to Boolean lattices.

Proposition 6.6. Let $B$ be an infinite Boolean lattice. Then $\operatorname{Id} B$ is not lower continuous.

Proof. Let $\left(a_{n}\right)_{n<\omega}$ be a strictly increasing sequence of elements of $B$. Let $A$ denote the ideal of $B$ generated by $\left\{a_{n} \mid n<\omega\right\}$ and let $B_{n}$ denote the principal ideal generated by $\neg a_{n}$, for all $n<\omega$. Observe that the sequence $\left(B_{n}\right)_{n<\omega}$ is (strictly) decreasing. Then the top element 1 belongs to $A \vee B_{n}$, for every $n<\omega$, but it does not belong to $A \vee \bigcap_{n<\omega} B_{n}$.

\section{DUAL $*$-DISTRIBUTIVITY}

Our $*$ operation is the dual of the one considered in [6] 19] and [8, Section 5.6].

Definition 7.1. For a lattice $L$, we consider the lattice $L \cup\{1\}$ obtained by adding a new largest element 1 to $L$. For $a \in L$, we define inductively an element $a * s$ of $L \cup\{1\}$, for $s \in L^{<\omega}$, as follows:

$$
\begin{aligned}
a * \varnothing & =1 ; \\
a *\left(s^{\frown\langle b\rangle)}\right) & =a \vee(b \wedge(a * s)), \text { for all } s \in L^{<\omega} \text { and } b \in L .
\end{aligned}
$$

For a subset $B$ of $L$, we put

$$
a * B=\left\{a * s \mid s \in B^{<\omega}\right\} .
$$

We say that $L$ is dually $*$-distributive, if whenever $a \in L$ and $B \subseteq L$, if $\bigwedge B$ exists, then $\bigwedge(a * B)$ exists and

$$
\bigwedge(a * B)=a \vee \bigwedge B .
$$

Restricting the cardinality of $B$ to be at most $\kappa$, for a given cardinal number $\kappa$, defines dual $\kappa$-*-distributivity (see Section 2). We say that $L$ is

- dually staircase distributive, if it is dually $n$-*-distributive, for every positive integer $n$, 
- dually zipper distributive, if it is dually 2 -*-distributive.

The following is essentially due to M. Erné, see the proof of [6] Proposition 2.12].

Proposition 7.2. Let $L$ be a lattice and let $\kappa$ be either an infinite cardinal number or $\infty$. Consider the following statements:

(i) $L$ is dually $\kappa$-*-distributive.

(ii) $L$ is $\kappa$-lower continuous and dually staircase distributive.

(iii) $L$ is $\kappa$-lower continuous and dually zipper distributive.

(iv) $L$ is $\kappa$-lower continuous and join-semidistributive.

Then (i) and (ii) are equivalent, they imply (iii), which implies (iv). Furthermore, if $L$ is $\kappa$-meet-complete, then all four statements are equivalent.

It will turn out that (i), (ii), and (iii) are, in fact, equivalent, see Corollary 11.4 The following fact is obvious.

Lemma 7.3. For a sublattice $K$ of a lattice $L$, the following statements hold.

(i) If $L$ is dually staircase distributive (resp., dually zipper distributive), then so is $K$.

(ii) If the inclusion map from $K$ into $L$ is meet-complete and $L$ is dually *distributive, then so is $K$.

The following result extends [8, Theorem 5.66].

Corollary 7.4. Every fermentable lattice is dually $*$-distributive.

Proof. It follows from Theorem 5.2 that $L$ has a meet-complete lattice embedding into some direct product $\bar{L}$ of finite lower bounded lattices. Of course, $\bar{L}$ is complete, lower continuous, and join-semidistributive, hence, by Proposition 7.2 it is dually *-distributive. Therefore, by Lemma 7.3 . $L$ is also dually $*$-distributive.

Corollary 7.5. Let $S$ be a join-semilattice satisfying the following assumptions:

(i) $S$ has the MCRP.

(ii) There are no infinite $D$-sequences in $\mathrm{J}(S)$.

Then $\operatorname{Id} S$ is dually *-distributive; in particular, it is join-semidistributive.

Proof. Apply Corollary 7.4 to $L=\operatorname{Id} S$ and $\Sigma=\{\downarrow p \mid p \in \mathrm{J}(S)\}$.

It is well-known that free lattices have the MCRP and have no infinite $D$ sequences, see [8, Chapter II]. Hence, we obtain the following consequence.

Corollary 7.6. Let $L$ be a lower bounded homomorphic image of a free lattice. Then $\operatorname{Id} L$ is dually $*$-distributive; in particular, it is join-semidistributive.

Compare with Corollary 6.5 Observe that we do not require $L$ to be finitely generated. By Corollary [5.4 Id $L$ satisfies many other quasi-identities than joinsemidistributivity, namely, all those quasi-identities that hold in all finite lower bounded lattices, see Theorem 4.2.8 and Corollary 5.5.8 in [12].

\section{The axiom $\left(\mathrm{SD}_{\vee}^{\omega}\right)$}

Let $L$ be a lattice. For any $s=\left\langle a_{0}, \ldots, a_{n-1}\right\rangle \in L^{<\omega}$, we put $n=|s|$, and, if $n>0$, we put $\boldsymbol{s}_{*}=\left\langle a_{0}, \ldots, a_{n-2}\right\rangle$ and $e(\boldsymbol{s})=a_{n-1}$. Furthermore, we define 
inductively $s \diamond x$, for $s \in L^{<\omega}$ and $x \in L$ :

$$
\begin{gathered}
\varnothing \diamond x=x ; \\
(s \frown\langle a\rangle) \diamond x= \begin{cases}a \vee(s \diamond x), & \text { if }|s| \text { is even, } \\
a \wedge(s \diamond x), & \text { if }|s| \text { is odd. }\end{cases}
\end{gathered}
$$

We shall need in Section 11 the following simple lemma.

Lemma 8.1. There are maps $j: L \times L^{<\omega} \rightarrow L^{<\omega}$ and $m: L \times L \times L^{<\omega} \rightarrow L^{<\omega}$ such that the following equalities hold for all $u, x, y \in L$ and all $s \in L^{<\omega}$ with $y \leq x$ :

$$
\begin{aligned}
& u \vee(s \diamond x)=j(u, s) \diamond x ; \\
& u \wedge(s \diamond x)=m(u, y, s) \diamond x .
\end{aligned}
$$

Proof. We define the maps $j$ and $m$ inductively, by

$$
\begin{aligned}
& j(u, \varnothing)=\langle u\rangle \quad \text { and } m(u, y, \varnothing)=\langle y, u\rangle \text {; } \\
& j(u, s)=\boldsymbol{s}^{\frown}\langle u\rangle \quad \text { and } m(u, y, \boldsymbol{s})=\boldsymbol{s}_{*} \frown\langle u \wedge e(\boldsymbol{s})\rangle \text {, if }|\boldsymbol{s}| \text { is nonzero even; } \\
& j(u, s)=\boldsymbol{s}_{*} \frown\langle u \vee e(\boldsymbol{s})\rangle \text { and } m(u, y, \boldsymbol{s})=\boldsymbol{s} \frown\langle u\rangle \text {, if }|\boldsymbol{s}| \text { is odd. }
\end{aligned}
$$

It is straightforward to verify that these maps satisfy the required conditions.

Definition 8.2. We say that a lattice $L$ satisfies $\left(\mathrm{SD}_{\vee}^{\omega}\right)$, if the following equality holds, for all $s \in L^{<\omega}$ and all $a, b, c \in L$ :

$$
s \diamond(a \vee(b \wedge c))=\bigwedge\left\{s \diamond(a * \boldsymbol{t}) \mid \boldsymbol{t} \in\{b, c\}^{<\omega}\right\} .
$$

It is not hard to verify that $\left(\mathrm{SD}_{\vee}^{\omega}\right)$ is a weakening of the identity $\left(\mathrm{SD}_{\vee}^{n}\right)$ considered in [15. Section 4.2]. A very closely related notion is the (dual) $m$-zipper distributivity considered in [6].

Proposition 8.3. Let $L$ be a lattice. Consider the following statements:

(i) L satisfies $\left(\mathrm{SD}_{\vee}^{\omega}\right)$.

(ii) $L$ is dually zipper distributive.

Then (i) implies (ii). Furthermore, if $L$ is $\aleph_{0}$-lower continuous, then (i) and (ii) are equivalent.

Proof. It is obvious that dual zipper distributivity of $L$ is equivalent to the satisfaction of (8.3) for all elements $a, b, c$ of $L$ and for $s=\varnothing$, and thus it follows from $\left(\mathrm{SD}_{\vee}^{\omega}\right)$. If $L$ is $\aleph_{0}$-lower continuous, then it is easy to establish, by induction on the length of $s$, the equality

$$
s \diamond \bigwedge X=\bigwedge\{s \diamond x \mid x \in X\},
$$

for all $s \in L^{<\omega}$ and every (at most) countable downward directed subset $X$ of $L$. Under such conditions, dual zipper distributivity obviously implies $\left(\mathrm{SD}_{\vee}^{\omega}\right)$.

Part of the conclusion of Proposition 8.3 will be strengthened in Corollary 11.3 .

We leave to the reader the easy proof of the following preservation result.

Proposition 8.4. The following statements hold.

(i) Every sublattice of a lattice satisfying $\left(\mathrm{SD}_{\vee}^{\omega}\right)$ satisfies $\left(\mathrm{SD}_{\vee}^{\omega}\right)$.

(ii) Every directed union of a family of lattices satisfying $\left(\mathrm{SD}_{\vee}^{\omega}\right)$ satisfies $\left(\mathrm{SD}_{\vee}^{\omega}\right)$.

(iii) Every image of a lattice satisfying $\left(\mathrm{SD}_{\vee}^{\omega}\right)$ under a $\aleph_{0}$-meet-complete lattice homomorphism satisfies $\left(\mathrm{SD}_{\vee}^{\omega}\right)$. 
(iv) Every lower bounded homomorphic image of a lattice satisfying $\left(\mathrm{SD}_{\vee}^{\omega}\right)$ satisfies $\left(\mathrm{SD}_{\vee}^{\omega}\right)$.

(Since every lower bounded surjective homomorphism is meet-complete, item (iii) trivially implies item (iv).)

As an immediate consequence of Proposition 8.4 (i,ii), we obtain the following.

Corollary 8.5. A lattice $L$ satisfies $\left(\mathrm{SD}_{\vee}^{\omega}\right)$ iff every finitely generated sublattice of $L$ satisfies $\left(\mathrm{SD}_{\vee}^{\omega}\right)$.

Corollary 8.6. Every directed union of fermentable lattices satisfies $\left(\mathrm{SD}_{\vee}^{\omega}\right)$.

Proof. By Corollary 7.4 every fermentable lattice is dually $*$-distributive, hence, by Propositions 7.2 and 8.3 it satisfies $\left(\mathrm{SD}_{\vee}^{\omega}\right)$. The conclusion follows from Proposition 8.4(ii).

\section{LOWER CONTINUOUS LATTICES OF FILTERS}

Definition 9.1. A notion of convergence on a lattice $L$ is a set $\mathbb{S}$ of subsets of $L$ satisfying the following conditions:

(S1) Every element $X$ of $\mathbb{S}$ is downward directed, furthermore, $\bigwedge X$ exists.

(S2) For all $X \in \mathbb{S}$ and all $a \in L$, the subset $a \vee X=\{a \vee x \mid x \in X\}$ belongs to $\mathbb{S}$, and $\bigwedge(a \vee X)=a \vee \wedge X$.

(S3) For all $X \in \mathbb{S}$ and all $a \in L$, the subset $a \wedge X=\{a \wedge x \mid x \in X\}$ belongs to $\mathbb{S}$. (Observe that necessarily, $\bigwedge(a \wedge X)=a \wedge \wedge X$.)

We say that $\mathbb{S}$ is special, if it satisfies the following condition, that involves the * operation introduced in Section $\mathbf{7}$

(S4) The subset $\left\{a * \boldsymbol{t} \mid \boldsymbol{t} \in\{b, c\}^{<\omega} \backslash\{\varnothing\}\right\}$ belongs to $\mathbb{S}$ and its meet is $a \vee(b \wedge$ $c)$, for all $a, b, c \in L$.

As usual, we say that a filter of a lattice $L$ is a (possibly empty) upper subset of $L$, closed under finite meets.

Definition 9.2. Let $\mathbb{S}$ be a set of subsets of a lattice $L$ satisfying (S1). We say that a subset $A$ of $L$ is $\mathbb{S}$-closed, if $X \subseteq A$ implies that $\bigwedge X \in A$, for all $X \in \mathbb{S}$.

For a subset $A$ of $L$, we denote by $\mathrm{Cl}_{\mathbb{S}}(A)$ the least $\mathbb{S}$-closed subset of $L$ containing $A$, and by $\mathrm{Fl}_{\mathbb{S}}(A)$ the least $\mathbb{S}$-closed filter containing $A$.

For the remainder of Section 9 , let $\mathbb{S}$ be a notion of convergence on a lattice $L$.

For any $a \in L$ and any $X \subseteq L$, we put

$$
\begin{aligned}
& X \nearrow a=\{y \in L \mid a \wedge y \in X\}, \\
& X \searrow a=\{y \in L \mid a \vee y \in X\} .
\end{aligned}
$$

The proof of the following lemma is a straightforward application of (S2) and (S3).

Lemma 9.3. If a subset $X$ of $L$ is $\mathbb{S}$-closed, then so are $X \nearrow$ a and $X \searrow a$, for any $a \in L$.

It is obvious that $\mathrm{Cl}_{\mathbb{S}}(A)$ is contained in $\mathrm{Fl}_{\mathbb{S}}(A)$, for any $A \subseteq L$. The following lemma gives us an important case where the two closures are equal.

Lemma 9.4. The equality $\mathrm{Cl}_{\mathbb{S}}(A)=\mathrm{Fl}_{\mathbb{S}}(A)$ holds, for any filter $A$ of $L$. 
F. WEHRUNG

Proof. Put $B=\mathrm{Cl}_{\mathbb{S}}(A)$. For any $a \in A$, the subset $B \nearrow a$ is, by Lemma $9.3 \mathbb{S}$ closed, and it contains $A$ (because $A$ is closed under finite meets); thus $B \subseteq B \nearrow a$. Hence $A \subseteq B \nearrow b$, for all $b \in B$. But $B \nearrow b$ is $\mathbb{S}$-closed, thus $B \subseteq B \nearrow b$, for all $b \in B$; that is, $B$ is closed under finite meets.

Furthermore, for any $x \in L$, the subset $B \searrow x$ is, by Lemma 9.3 S-closed, but it contains $A$ (because $A$ is an upper subset of $L$ ), thus it contains $B$. Hence $B$ is an upper subset of $L$. Therefore, $B$ is a filter of $L$, but it is $\mathbb{S}$-closed, thus it contains $\mathrm{Fl}_{\mathbb{S}}(A)$; whence $B=\mathrm{Fl}_{\mathbb{S}}(A)$.

We denote by $\mathrm{Fil}^{\mathbb{S}} L$ the set of all $\mathbb{S}$-closed filters $X$ of $L$ such that if $L$ has a unit element, say, $1_{L}$, then $1_{L} \in X$. (We take this precaution in order to ensure that the canonical embedding from $\langle L, \leq\rangle$ into $\left\langle\mathrm{Fil}^{\mathbb{S}} L, \supseteq\right\rangle$ preserves the empty meet.) Since $\mathrm{Fil}^{\mathbb{S}} L$ is a closure system in the powerset of $L$, the poset $\left\langle\mathrm{Fil}^{\mathbb{S}} L, \subseteq\right\rangle$ is a complete lattice. We shall order $\mathrm{Fil}^{\mathbb{S}} L$ by reverse inclusion. The proof of the following lemma is obvious.

Lemma 9.5. The map $x \mapsto \uparrow x$ defines a join-complete lattice embedding from $\langle L, \leq\rangle$ into $\left\langle\mathrm{Fil}^{\mathbb{S}} L, \leq\right\rangle$.

The following result is much less obvious.

Proposition 9.6. The lattice $\left\langle\mathrm{Fil}^{\mathbb{S}} L, \leq\right\rangle$ is lower continuous.

Proof. We prove that the dual lattice $\left\langle\mathrm{Fil}^{\mathbb{S}} L, \subseteq\right\rangle$ is upper continuous. We put $\bigvee_{i \in I}^{*} X_{i}=\mathrm{Fl}_{\mathbb{S}}\left(\bigcup_{i \in I} X_{i}\right)$, for any family $\left(X_{i}\right)_{i \in I}$ of elements of $\mathrm{Fil}^{\mathbb{S}} L$, so it suffices to prove the containment

$$
A \cap \bigvee_{i \in I}^{*} B_{i} \subseteq \bigvee_{i \in I}^{*}\left(A \cap B_{i}\right)
$$

for any $A \in \mathrm{Fil}^{\mathbb{S}} L$, any upper directed poset $I$, and any increasing (for the inclusion) family $\left(B_{i}\right)_{i \in I}$ of elements of $\mathrm{Fil}^{\mathbb{S}} L$. Put $B=\bigcup_{i \in I} B_{i}$, observe that $B$ is a filter of $L$; thus, by Lemma $9.4 \mathrm{Cl}_{\mathbb{S}}(B)=\bigvee_{i \in I}^{*} B_{i}$. Denote by $C$ the right hand side of (9.3), and put

$$
D=\bigcap_{a \in A}(C \searrow a) \text {. }
$$

For all $i \in I$ and $b \in B_{i}$, the element $a \vee b$ belongs to $A \cap B_{i}$, thus to $C$; whence $B \subseteq D$. It follows from Lemma 9.3 that $D$ is $\mathbb{S}$-closed, thus $\mathrm{Cl}_{\mathbb{S}}(B) \subseteq D$. This means that $a \vee b \in C$, for all $a \in A$ and all $b \in \mathrm{Cl}_{\mathbb{S}}(B)=\bigvee_{i \in I}^{*} B_{i}$, which concludes the proof of (9.3).

\section{JoIN-SEMIDISTRIBUTIVE LATTICES OF FILTERS}

The main result of the present section invokes the special notions of convergence introduced in Definition 9.1

Proposition 10.1. Let $\mathbb{S}$ be a special notion of convergence on a lattice $L$. Then the lattice $\mathrm{Fil}^{\mathbb{S}} L$ (with reverse inclusion) is complete, lower continuous, and joinsemidistributive.

Proof. We have already observed that $\mathrm{Fil}^{\mathbb{S}} L$ is complete, and, by Proposition 9.6 lower continuous. In order to prove that $\mathrm{Fil}^{\mathbb{S}} L$ is join-semidistributive, it suffices to prove that for all $A, B, C, D \in \mathrm{Fil}^{\mathbb{S}} L$ such that $A \cap B=A \cap C=D$, the 
containment $A \cap\left(B \vee^{*} C\right) \subseteq D$ holds, where we put $B \vee^{*} C=\operatorname{Fl}_{\mathbb{S}}(B \cup C)$. Let $F$ denote the filter of $L$ generated by $B \cup C$.

We prove that $a \vee x \in D$, for all $\langle a, x\rangle \in A \times F$. Let $\langle b, c\rangle \in B \times C$ such that $b \wedge c \leq x$. We prove, by induction on $|s|$, that $a * s \in D$, for all $s \in\{b, c\}^{<\omega} \backslash\{\varnothing\}$. For $\boldsymbol{s}=\langle b\rangle$, we have $a * \boldsymbol{s}=a \vee b \in D$, and similarly for $\boldsymbol{s}=\langle c\rangle$. Suppose that $|\boldsymbol{s}|>1$. It follows from the induction hypothesis that $a * s_{*} \in D$, thus $e(s) \wedge\left(a * s_{*}\right) \in B \cup C$, and therefore

$$
a * s=a \vee\left(e(s) \wedge\left(a * \boldsymbol{s}_{*}\right)\right) \in D .
$$

Since the set $D$ is $\mathbb{S}$-closed and $\mathbb{S}$ is a special notion of convergence, $a \vee(b \wedge c)=$ $\bigwedge\left(a * s \mid s \in\{b, c\}^{<\omega}\right)$ belongs to $D$. Since $D$ is an upper subset of $L$, it follows that $a \vee x \in D$. Hence we have proved the containment $F \subseteq E$, where we put

$$
E=\bigcap(D \searrow a \mid a \in A) .
$$

Since $E$ is $\mathbb{S}$-closed, we conclude, by using Lemma 9.4 that $B \vee^{*} C=\mathrm{Fl}_{\mathbb{S}}(F)=$ $\mathrm{Cl}_{\mathbb{S}}(F) \subseteq E$. This means that $a \vee x \in D$, for all $a \in A$ and all $x \in B \vee^{*} C$.

We immediately obtain the following corollary, see [6, Proposition 2.18]. Compare with Example 11.10

Corollary 10.2. The filter lattice Fil $L$ of every locally finite, join-semidistributive lattice $L$ is join-semidistributive.

Proof. Let $\mathbb{S}$ denote the set of all finite subsets of $L$ with a least element. It is obvious that $\mathbb{S}$ is a special notion of convergence on $L$, furthermore $\mathrm{Fil}^{\mathbb{S}} L=$ Fil $L$ is a dually algebraic lattice. The conclusion follows from Proposition 10.1

We recall that there exists a join-semidistributive lattice that cannot be embedded into any complete, join-semidistributive lattice, see [4 Example 3.25]. Whether or not such a lattice can be taken finitely generated is apparently an open problem, see [4. Problem 2].

\section{Sublattices of COMPlete, lower Continuous, Join-Semidistributive} LATTICES

In this section we shall reap the consequences of Sections 8 10 We first prove a simple lemma.

Lemma 11.1. Let $\kappa$ be either an infinite cardinal or $\infty$. Let $K$ be a sublattice of a $\kappa$-lower continuous lattice $L$. Denote by $K^{\prime}$ the set of all meets of downward directed subsets of $K$ with at most $\kappa$ elements. Then $K^{\prime}$ is a sublattice of $L$, and it belongs to the same variety as $K$.

Proof. The fact that $K^{\prime}$ is a sublattice of $L$ follows from the continuity assumption on $L$. Now let $m>0$ and let $\mathbf{s}$ and $\mathbf{t}$ be lattice terms with $m$ variables such that $K$ satisfies the identity $\mathbf{s}=\mathbf{t}$. Let $a_{0}, \ldots, a_{m-1} \in K^{\prime}$. For all $i<m$, there exists a downward directed subset $X_{i}$ of $K$ with at most $\kappa$ elements such that $a_{i}=\bigwedge X_{i}$. By using the continuity assumption on $L$, we obtain:

$$
\begin{aligned}
\mathbf{s}\left(a_{0}, \ldots, a_{m-1}\right) & =\bigwedge\left(\mathbf{s}\left(x_{0}, \ldots, x_{m-1}\right) \mid x_{i} \in X_{i}, \text { for all } i<m\right) \\
& =\bigwedge\left(\mathbf{t}\left(x_{0}, \ldots, x_{m-1}\right) \mid x_{i} \in X_{i}, \text { for all } i<m\right) \\
& =\mathbf{t}\left(a_{0}, \ldots, a_{m-1}\right),
\end{aligned}
$$

which proves that $K^{\prime}$ satisfies the identity $\mathbf{s}=\mathbf{t}$. 
Theorem 11.2. For any lattice $L$, the following statements are equivalent:

(i) L has a join-complete lattice embedding into some complete, lower continuous, join-semidistributive lattice that belongs to the same variety as $L$.

(ii) L has a lattice embedding into some complete, lower continuous, join-semidistributive lattice.

(iii) $L$ has a lattice embedding into some $\aleph_{0}$-meet-complete, $\aleph_{0}$-lower continuous, join-semidistributive lattice.

(iv) L satisfies the axiom $\left(\mathrm{SD}_{\vee}^{\omega}\right)$.

Proof. (i) $\Rightarrow$ (ii) and (ii) $\Rightarrow$ (iii) are trivial.

(iii) $\Rightarrow$ (iv) follows immediately from Propositions 7.28 .3 and 8.4 (i).

(iv) $\Rightarrow$ (i) Let $L$ be a lattice satisfying $\left(\mathrm{SD}_{\vee}^{\omega}\right)$. We put

$$
U(\boldsymbol{s} ; a, b, c)=\left\{\boldsymbol{s} \diamond(a * \boldsymbol{t}) \mid \boldsymbol{t} \in\{b, c\}^{<\omega} \backslash\{\varnothing\}\right\},
$$

for all $a, b, c \in L$ and all $s \in L^{<\omega}$. Furthermore, we put

$$
\mathbb{S}=\left\{U(s ; a, b, c) \mid a, b, c \in L \text { and } s \in L^{<\omega}\right\} .
$$

It follows from the assumption ( $\left.\mathrm{SD}_{\vee}^{\omega}\right)$ that $\mathbb{S}$ satisfies (S1). Let $X \in \mathbb{S}$ and $u \in L$. Write $X=U(s ; a, b, c)$, for some $a, b, c \in L$ and $s \in L^{<\omega}$. It follows from Lemma 8.1 that $u \vee X=U(j(u, s) ; a, b, c)$ belongs to $\mathbb{S}$, whence $u \vee X$ has a meet in $L$, and consequently, by using $\left(\mathrm{SD}_{\vee}^{\omega}\right)$ and Lemma 8.1

$$
\bigwedge(u \vee X)=j(u, s) \diamond(a \vee(b \wedge c))=u \vee(s \diamond(a \vee(b \wedge c)))=u \vee \bigwedge X
$$

On the other hand, observe that $b \wedge c \leq a * \boldsymbol{t}$, for all $\boldsymbol{t} \in\{b, c\}^{<\omega}$, whence $u \wedge X=$ $U(m(u, b \wedge c, s) ; a, b, c)$ belongs to $\mathbb{S}$. Therefore, $\mathbb{S}$ satisfies (S2) and (S3). Finally, $\left\{a * \boldsymbol{t} \mid \boldsymbol{t} \in\{b, c\}^{<\omega} \backslash\{\varnothing\}\right\}$ is equal to $U(\varnothing ; a, b, c)$, thus it belongs to $\mathbb{S}$ and its meet is $a \vee(b \wedge c)$, and hence $\mathbb{S}$ is a special notion of convergence on $L$.

By Lemma $9.5 L$ has a join-complete lattice embedding into $\widehat{L}=\mathrm{Fil}^{\mathbb{S}} L$. By the paragraph above and by Proposition 10.1 $\widehat{L}$ is complete, lower continuous, and join-semidistributive. For a subset $X$ of $\widehat{L}$, let $X^{\downarrow \wedge}$ denote the set of all meets of downwards directed subsets of $X$. Put $L_{0}=L, L_{\xi+1}=\left(L_{\xi}\right)^{\downarrow \wedge}$ for any ordinal $\xi$, and $L_{\lambda}=\bigcup_{\xi<\lambda} L_{\xi}$ for any limit ordinal $\lambda$. It follows from Lemma 11.1 that $L_{\xi}$ is a sublattice of $\widehat{L}$ (thus it is join-semidistributive), and it belongs to the same variety as $L$, for any ordinal $\xi$. Hence the same holds for $L^{*}=\bigcup_{\xi} L_{\xi}$. Furthermore, $L^{*}$ is a complete meet-subsemilattice of $\widehat{L}$. Therefore, $L^{*}$ is complete, lower continuous, join-semidistributive, and the inclusion map from $L$ into $L^{*}$ is join-complete.

Corollary 11.3. Every lattice satisfying $\left(\mathrm{SD}_{\vee}^{\omega}\right)$ is dually staircase $*$-distributive.

Proof. Let $L$ be a lattice satisfying $\left(\mathrm{SD}_{\vee}^{\omega}\right)$. It follows from Theorem 11.2 that $L$ can be embedded into some complete, lower continuous, and join-semidistributive lattice $\bar{L}$. It follows from Proposition 7.2 that $\bar{L}$ is dually staircase distributive. Hence, by Lemma $7.3 L$ is also dually staircase distributive.

Hence, by using Proposition 8.3 we obtain the following.

Corollary 11.4. Let $L$ be $a \aleph_{0}$-lower continuous lattice. If $L$ is dually zipper distributive, then $L$ is dually staircase distributive. 
We emphasize that we do not assume completeness of $L$ in the statements of Corollaries 11.3 and 11.4

By using Corollary 8.6 we obtain the following.

Corollary 11.5. Every lower bounded lattice can be embedded into some complete, lower continuous, join-semidistributive lattice.

One cannot hope to strengthen the conclusion of Corollary 11.5 by requiring the larger lattice to be lower bounded; see also [2, p. 207]. The proof of the following lemma is straightforward, and left to the reader.

Lemma 11.6. Let $K$ be a sublattice of a lattice $L$. We assume that the inclusion map from $K$ into $L$ is lower bounded. If a nonempty subset $X$ of $K$ has a meet in $L$, then $X$ has a meet in $K$, and the two meets are equal.

Example 11.7. The free lattice on three generators $\mathrm{F}_{\mathbf{L}}(3)$ cannot be embedded into any $\aleph_{0}$-meet-complete, lower bounded lattice.

Proof. Suppose that $\mathrm{F}_{\mathbf{L}}(3)$ is a sublattice of a $\aleph_{0}$-meet-complete, lower bounded lattice $L$. Since $\mathrm{F}_{\mathbf{L}}(3)$ is finitely generated and $L$ is lower bounded, the inclusion $\operatorname{map} f: \mathrm{F}_{\mathbf{L}}(3) \hookrightarrow L$ is lower bounded. Hence, it follows from Lemma11.6 that $\mathrm{F}_{\mathbf{L}}(3)$ is $\aleph_{0}$-meet-complete, which is known not to be the case (see [8, Section I.5]).

It is noteworthy to record the following immediate consequence of Proposition 8.4 Corollary 8.5 and Theorem 11.2

Corollary 11.8. A lattice $L$ has an embedding into some complete, lower continuous, join-semidistributive lattice iff every finitely generated sublattice of $L$ has such an embedding.

The following example is the lower part of Example 3.25 in 4], and it is also the dual, minus the top element, of the lattice $Z_{\omega}$ of [6, Page 299]. It shows that lower continuity cannot be replaced by upper continuity in the statement of Corollary 11.5 .

Example 11.9. The lattice $L$ of Figure 1 is locally finite and lower bounded, but it cannot be embedded into any $\aleph_{0}$-join-complete, $\aleph_{0}$-upper continuous, join-semidistributive lattice.

Proof. It is straightforward to verify that the lattice $L$ is locally finite and lower bounded. Let $L^{\prime}$ be a $\aleph_{0}$-join-complete, $\aleph_{0}$-upper continuous, join-semidistributive lattice containing $L$. Put $a=\bigvee_{n<\omega} a_{n}$ and $b=\bigvee_{n<\omega} b_{n}$. Since $a_{n} \leq b_{n} \vee c$ and $b_{n} \leq$ $a_{n+1} \vee c$, for all $n<\omega$, the equality $a \vee c=b \vee c$ holds. Since $L^{\prime}$ is join-semidistributive, the inequality $a \leq(a \wedge b) \vee c$ holds, thus, a fortiori, $a_{1} \leq(a \wedge b) \vee c$. However, since $L^{\prime}$ is $\aleph_{0}$-join complete and $\aleph_{0}$-upper continuous, $a \wedge b=\bigvee_{n<\omega}\left(a_{n} \wedge b_{n}\right)=0$, so we obtain the inequality $a_{1} \leq c$, a contradiction.

On the other hand, the duals of [6] Proposition 2.18] and [19 Lemma 10] imply immediately the following example. It illustrates the importance of the choice of $\mathbb{S}$ in the proof of Corollary 11.5 Compare also with Corollaries 7.6 and 10.2

Example 11.10. The filter lattice $L$ of $\mathrm{F}_{\mathbf{L}}(3)$ is not join-semidistributive.

We conclude this section by the following analogue of Theorem 11.2 for complete embeddings. 


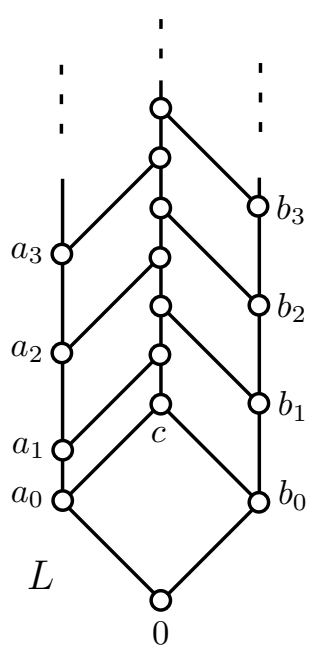

Figure 1. A locally finite lower bounded lattice.

Theorem 11.11. For any lattice $L$, the following statements are equivalent:

(i) L has a complete lattice embedding into some complete, lower continuous, join-semidistributive lattice.

(ii) L has a meet-complete lattice embedding into some complete, lower continuous, join-semidistributive lattice.

(iii) $L$ is dually $*$-distributive.

(iv) $L$ is dually zipper distributive and lower continuous.

Proof. (i) $\Rightarrow$ (ii) is trivial.

(ii) $\Rightarrow$ (iii) follows immediately from Proposition 7.2 and Lemma 7.3

(iii) $\Rightarrow$ (iv) follows immediately from Proposition 7.2

(iv) $\Rightarrow$ (i). Let $L$ be dually zipper distributive and lower continuous. We put

$$
\mathbb{S}=\{X \subseteq L \mid X \text { is downward directed and } \bigwedge X \text { exists }\}
$$

It is straightforward to verify that $\mathbb{S}$ is a special notion of convergence on $L$ and that the map $x \mapsto \uparrow x$ is a complete lattice embedding from $L$ into $\mathrm{Fil}^{\mathbb{S}} L$. By Proposition 10.1 $\mathrm{Fil}^{\mathbb{S}} L$ is complete, lower continuous, and join-semidistributive.

Most classes of lattices encountered in the present paper are represented on Figure 2. The largest classes are on the top of the diagram. The diagram without $(\Phi)$ (i.e., fermentability) is a meet-semilattice, for example, the intersection of $\left(\mathrm{SD}_{\vee}\right)$ and $\left(\mathrm{LC}^{+}\right)$is, indeed, contained in the class $\left(\mathrm{D}^{*} \mathrm{D}\right)$ of all dually $*$-distributive lattices.

\section{Non-Embeddability RESUlts into BI-ALGEBRAiC LATTiCES}

We first state the central lemma underlying all the results of the present section.

Lemma 12.1. Let $L$ be $a \aleph_{0}$-complete, $\aleph_{0}$-upper continuous, and $\aleph_{0}$-lower continuous lattice with zero. Let $\left(a_{n}\right)_{n<\omega}$ be a sequence of elements of $L$ and let $c \in L$ such that the following statements hold: 


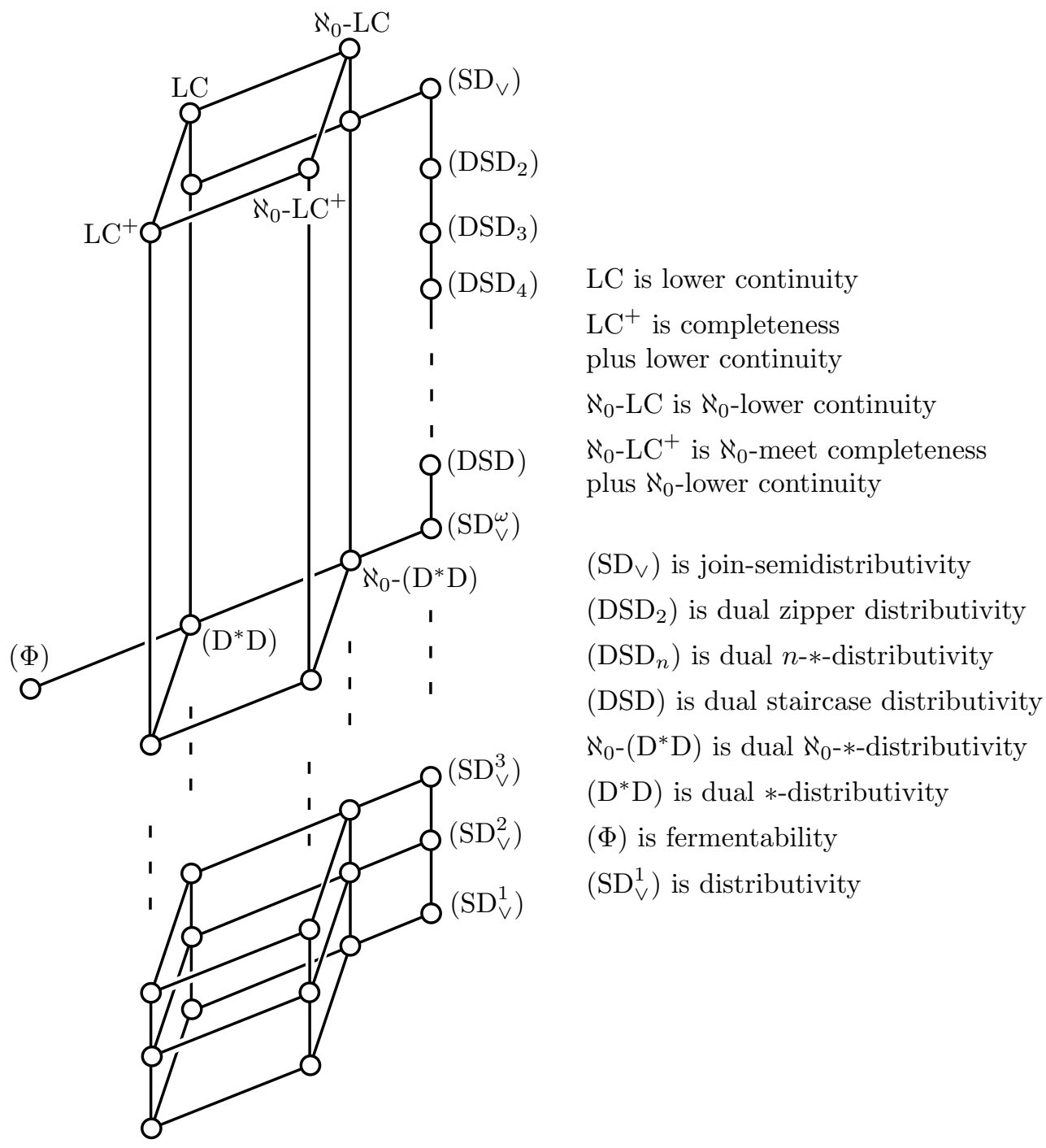

Figure 2. Classes of lattices.

(i) $\left(\bigvee_{i<m} a_{i}\right) \wedge\left(\bigvee_{j<\omega} a_{m+j}\right)=0$, for all $m<\omega$.

(ii) $a_{0} \leq \bigvee_{j<\omega} a_{m+j} \vee c$, for all $m<\omega$.

(iii) $a_{0} \wedge c=0$.

Then $a_{0}=0$.

Proof. Put $b_{n}=\bigvee_{j<\omega} a_{n+j}$, for all $n<\omega$, and $b=\bigwedge_{n<\omega} b_{n}$. It follows from (i) that $\left(\bigvee_{i<n} a_{i}\right) \wedge b=0$, for all $n<\omega$, hence, by the $\aleph_{0}$-upper continuity of $L$, we obtain that $b=0$. Since (ii) can be written $a_{0} \leq b_{m} \vee c$ for all $m<\omega$, it follows from the $\aleph_{0}$-lower continuity of $L$ that $a_{0} \leq b \vee c=c$. Therefore, from (iii) it follows that $a_{0}=0$. 
Corollary 12.2. Let $L$ be a lattice with zero, let $\left(a_{n}\right)_{n<\omega}$ be a sequence of elements of $L$, and let $c \in L$ such that $a_{0} \neq 0$ and the following statements hold:

(i) $\left(\bigvee_{i<m} a_{i}\right) \wedge\left(\bigvee_{j<n} a_{m+j}\right)=0$, for all $m, n<\omega$.

(ii) For all $m<\omega$, there exists $n<\omega$ such that $a_{0} \leq \bigvee_{j<n} a_{m+j} \vee c$.

(iii) $a_{0} \wedge c=0$.

Then $L$ cannot be embedded into any $\aleph_{0}$-complete, $\aleph_{0}$-upper continuous, and $\aleph_{0}$-lower continuous lattice.

Proof. Let $L^{\prime}$ be a $\aleph_{0}$-complete, $\aleph_{0}$-upper continuous, and $\aleph_{0}$-lower continuous lattice such that $L$ embeds into $L^{\prime}$. After replacing $L^{\prime}$ by $\uparrow L$, we may assume, without loss of generality, that $L^{\prime}$ has the same zero as $L$. It is trivial that (ii) and (iii) of the statement of Corollary 12.2 imply, respectively, (ii) and (iii) of the statement of Lemma 12.1 Since $L^{\prime}$ is $\aleph_{0}$-upper continuous, (i) follows as well. By Lemma 12.1 $a_{0}=0$, a contradiction.

As usual, in any modular lattice $L$ with zero, let $c=a \oplus b$ mean that $c=a \vee b$ while $a \wedge b=0$. A family $\left(a_{i}\right)_{i \in I}$ of elements of $L$ is independent, if the equality

$$
\left(\bigvee\left(a_{i} \mid i \in X\right)\right) \wedge\left(\bigvee\left(a_{i} \mid i \in Y\right)\right)=\bigvee\left(a_{i} \mid i \in X \cap Y\right)
$$

holds, for all finite subsets $X$ and $Y$ of $I$. Since $L$ is modular, it is sufficient to verify this for $X$ a singleton, see [13. We say that $a, b \in L$ are perspective, if there exists $c \in L$ such that $a \oplus c=b \oplus c$.

Corollary 12.3. Let $L$ be a modular lattice with zero, suppose that $L$ has an infinite independent sequence of nonzero pairwise perspective elements. Then $L$ cannot be embedded into any $\aleph_{0}$-complete, $\aleph_{0}$-upper continuous, and $\aleph_{0}$-lower continuous lattice.

Proof. Suppose that $L$ embeds into a $\aleph_{0}$-complete, $\aleph_{0}$-upper continuous, and $\aleph_{0}$ lower continuous lattice $L^{\prime}$. As above, we may assume that $L$ and $L^{\prime}$ have the same zero. Now we use Lemma 11.1 (and its dual) as in the proof of (iv) $\Rightarrow$ (i) of Theorem 11.2 but this time by alternating $\omega_{1}$ times closure under countable meets and joins. We obtain that the closure $L^{*}$ of $L$ (within $L^{\prime}$ ) under countable meets and countable joins belongs to the same variety as $L$. In particular, in addition to being $\aleph_{0}$-complete, $\aleph_{0}$-upper continuous, and $\aleph_{0}$-lower continuous, the lattice $L^{*}$ is modular.

Let $\left(a_{n}\right)_{n<\omega}$ be a sequence of pairwise perspective elements of $L$, with $a_{0} \neq 0$. For all $n>0$, there exists $c_{n} \in L$ such that $a_{0} \oplus c_{n}=a_{n} \oplus c_{n}=a_{0} \vee a_{n}$. It is an easy exercise to verify that the $a_{n}$-s and $c=\bigvee_{n>0} c_{n}$ satisfy the assumptions of Corollary 12.2 (with $n=1$ in (ii)), see also the proof of [17, Theorem I.3.8] or [16, Satz IV.2.1]. Hence, by Corollary [12.2 $a_{0}=0$, a contradiction.

Corollary 12.4. Let $V$ be an infinite-dimensional vector space over a division ring. Then the subspace lattice of $V$ cannot be embedded into any $\aleph_{0}$-complete, $\aleph_{0}$-upper continuous, and $\aleph_{0}$-lower continuous lattice.

Corollary 12.5. Let $\langle I, \unlhd\rangle$ be an infinite chain. Then the lattice $\mathbf{C o}(I)$ of orderconvex subsets of $I$ cannot be embedded into any $\aleph_{0}$-complete, $\aleph_{0}$-upper continuous, and $\aleph_{0}$-lower continuous lattice. 
Proof. Without loss of generality, $I$ has an infinite, strictly increasing sequence $z \triangleleft x_{0} \triangleleft x_{1} \triangleleft x_{2} \triangleleft \cdots$. Put $a_{m}=\left\{x_{m}\right\}$, for all $m<\omega$, and $c=\{z\}$. It is obvious that the $a_{m}$-s and $c$ satisfy the assumptions (i), (ii) (for $n=1$ ), and (iii) of Corollary 12.2 The conclusion follows from Corollary 12.2

Both Corollaries 12.4 and 12.5 solve negatively Problem 5 of [21].

\section{Open PROBLEMS}

Figure 2 shows containments between various classes of join-semidistributive lattices. This suggests the following general problem.

Problem 1. Do all lines on Figure 2 represent proper containments?

Of course, some partial answers to Problem 1 are known. For example, the classes $\left(\mathrm{SD}_{\vee}^{n}\right)$ and $\left(\mathrm{SD}_{\vee}^{n+1}\right)$ are, indeed, distinct, for every positive integer $n$. Many related examples can also be found in [6]. On the other hand, we do not have any example to show that dual staircase distributivity and dual zipper distributivity are really distinct notions - they coincide in the presence of $\aleph_{0}$-lower continuity, see Corollary 11.4 and Figure 2. Also observe that a lattice $L$ is join-semidistributive iff every three-generated sublattice of $L$ is join-semidistributive. A related problem, inspired by Corollary 11.8 is the following.

Problem 2. Does there exist a positive integer $n$ such that a lattice $L$ satisfies $\left(\mathrm{SD}_{\vee}^{\omega}\right)$ iff every $n$-generated sublattice of $L$ satisfies $\left(\mathrm{SD}_{\vee}^{\omega}\right)$ ?

It follows from Corollary 11.5 that every lower bounded lattice can be embedded into some complete, lower continuous, join-semidistributive lattice. The result would look better if we could replace "lower continuous" by "dually algebraic", but this we do not know.

Problem 3. Can every lower bounded lattice be embedded into some dually algebraic join-semidistributive lattice?

Trying to improve the universal theory instead of the completeness condition yields, for example, the following problem.

Problem 4. Can every lower bounded lattice be embedded into some complete, lower continuous lattice in $\mathbf{Q}\left(\mathcal{L B}_{\text {fin }}\right)$ ?

It is conceivable that the extension $\mathrm{Fil}^{\mathbb{S}} L$ defined in the proof of Corollary 11.5 belongs to $\mathbf{Q}\left(\mathcal{L B}_{\text {fin }}\right)$, but we do not know how to prove this.

It follows from Whitman's Theorem that every lattice can be embedded into an algebraic and spatial lattice, namely, a partition lattice. It is also proved in 14] that every modular lattice can be embedded, within its variety, into an algebraic and spatial lattice.

Problem 5. Can every lattice be embedded, within its variety, into some algebraic and spatial lattice?

For every algebraic lattice $A$, the lattice $\mathbf{S}_{\mathbf{p}}(A)$ of all algebraic subsets of $A$ (see [11, 12]) is dually algebraic and join-semidistributive.

Problem 6. Can every complete, lower continuous (resp., dually algebraic), joinsemidistributive lattice be embedded into $\mathbf{S}_{\mathbf{p}}(A)$, for some complete, upper continuous (resp., algebraic) lattice $A$ ? 
The deepest result of [4] is probably that every finite join-semidistributive lattice can be embedded into $\mathbf{S}_{\mathbf{p}}(A)$, for some algebraic lattice $A$.

\section{ACKNOWLEDGMENT}

I thank the anonymous referee for his careful reading of the paper, which lead to the correction of several embarrassing oversights.

\section{REFERENCES}

[1] M.E. Adams and W. Dziobiak, Q-universal quasivarieties of algebras, Proc. Amer. Math. Soc. 120, no. 4 (1994), 1053-1059.

[2] K. V. Adaricheva and V. A. Gorbunov, On lower bounded lattices, Algebra Universalis 46, no. $1 / 2$ (2001), 203-213.

[3] K. V. Adaricheva, V. A. Gorbunov, and M. V. Semenova, On continuous noncomplete lattices, Algebra Universalis 46, no. 1/2 (2001), 215-230.

[4] K. V. Adaricheva, V.A. Gorbunov, and V. I. Tumanov, Join-semidistributive lattices and convex geometries, Adv. Math. 173 (2003), 1-49.

[5] P. Crawley and R. P. Dilworth, "Algebraic Theory of Lattices", Prentice-Hall, New Jersey, 1973. vi+201 p.

[6] M. Erné, Weak distributive laws and their role in lattices of congruences of equational theories, Algebra Universalis 25, no. 3 (1988), 290-321.

[7] R. Freese, Finitely presented lattices: canonical forms and the covering relation, Trans. Amer. Math. Soc. 312, no. 1 (1989), 841-860.

[8] R. Freese, J. Ježek, and J.B. Nation, "Free Lattices", Mathematical Surveys and Monographs 42, Amer. Math. Soc., Providence, 1995. viii+293 p.

[9] R. Freese and J. B. Nation, Congruence lattices of semilattices, Pacific J. Math. 49, no. 1 (1973), 51-58.

[10] G. Gierz, K. H. Hofmann, K. Keimel, J. D. Lawson, M. Mislove, and D. S. Scott, "A Compendium of Continuous Lattices", Springer-Verlag, Berlin, New York, 1980, xx+371 p

[11] V.A. Gorbunov, The structure of lattices of quasivarieties, Algebra Universalis 32 (1994), 493-530.

[12] V.A. Gorbunov, "Algebraic theory of quasivarieties", (Algebraicheskaya teoriya kvazimnogoobrazij) (Russian) Sibirskaya Shkola Algebry i Logiki. 5. Novosibirsk: Nauchnaya Kniga, 1999. xii +368 p. English translation by Plenum, New York, 1998. xii+298 p.

[13] G. Grätzer, "General Lattice Theory. Second edition", new appendices by the author with B. A. Davey, R. Freese, B. Ganter, M. Greferath, P. Jipsen, H. A. Priestley, H. Rose, E. T. Schmidt, S. E. Schmidt, F. Wehrung, and R. Wille. Birkhäuser Verlag, Basel, 1998. xx+663 p.

[14] C. Herrmann, D. Pickering, and M. Roddy, A geometric description of modular lattices, Algebra Universalis 31, no. 3 (1994), 365-396.

[15] P. Jipsen and H. Rose, "Varieties of Lattices", Lecture Notes in Mathematics 1533, SpringerVerlag, Berlin Heidelberg, 1992. x+162 p.

[16] F. Maeda, "Kontinuierliche Geometrien" (German), die Grundlehren der mathematischen Wissenschaften in Einzeldarstellungen mit besonderer Berücksichtigung der Anwendungsgebiete, Bd. 95. Springer-Verlag, Berlin-Göttingen-Heidelberg, 1958. x+244 p. (translated from Japanese by S. Crampe, G. Pickert and R. Schauffler).

[17] J. von Neumann, "Continuous geometry", foreword by Israel Halperin. Princeton Mathematical Series, Vol. 25, Princeton University Press, Princeton, N. J., 1960. xi+299 p.

[18] P. Pudlák and J. Tůma, Yeast graphs and fermentation of algebraic lattices, Colloq. Math. Soc. János Bolyai 14, Lattice Theory, Szeged, 1974.

[19] J. Reinhold, Weak distributive laws and their role in free lattices, Algebra Universalis 33, no. 2 (1995), 209-215.

[20] M. V. Semenova, Sublattices of suborder lattices, preprint 2002.

[21] M. Semenova and F. Wehrung, Sublattices of lattices of order-convex sets, III. The case of totally ordered sets, Internat. J. Algebra Comput., to appear.

[22] F. Wehrung and M.V. Semenova, Sublattices of lattices of convex subsets of vector spaces, Algebra and Logic, to appear. 
LMnO, CNRS UMR 6139, Université de Caen, Campus II, Département de Mathématiques, B.P. 5186, 14032 CAEN CEDEx, FRANCE

E-mail address: wehrung@math.unicaen.fr

URL: http://www.math.unicaen.fr/ ${ }^{\sim}$ wehrung 